\title{
ACTITUDES Y CREENCIAS DE LOS DOCENTES HACIA EL ALUMNADO CON ALTAS CAPACIDADES INTELECTUALES Y LA IMPORTANCIA DE LA FORMACIÓN DEL PROFESORADO EN ESTE ÁMBITO
}

\author{
Carmen Gómez Labrador \\ carmengomezlab@gmail.com
}

Recepción Artículo: 22 octubre 2021 Admisión Evaluación: 22 octubre 2021 Informe Evaluador 1: 23 octubre 2021 Informe Evaluador 2: 24 octubre 2021 Aprobación Publicación: 24 octubre 2021

\section{RESUMEN}

Esta investigación plantea demostrar cómo la formación específica que reciben los docentes, en este caso, sobre el alumnado con altas capacidades intelectuales, es capaz de modificar favorablemente, creencias y actitudes previas hacia estos alumnos. Numerosos estudios consultados mundialmente, avalan que, entender cómo son estos alumnos y cuáles son sus necesidades, ayuda al profesorado a mejorar las actitudes hacia ellos y sus posibilidades de respuesta educativa. En este estudio, utilizando la escala Opinions about the gifted and their education, original de Gagné y Nadeau (1991), traducida y adaptada al español, se han comparado cómo son las actitudes previas en los participantes, un grupo experimental de 167 docentes de todas las etapas educativas, con los que se realizó un diseño cuasi-experimental, pretest-postest.

Los resultados indicaron que efectivamente, tras esta formación se modificaron favorablemente algunas actitudes que presentaban inicialmente los docentes, observándose una mejora en cuanto a la opinión subjetiva que tienen de este colectivo, hacia el rechazo que se le supone inicialmente a estos alumnos y su dificultad para tener buenas relaciones sociales y sobre todo en la consideración de medidas educativas con este alumnado, mostrándose más predispuestos hacia la aceleración y flexibilización de los alumnos con altas capacidades en el ámbito escolar, considerando necesaria la inclusión educativa del alumnado con altas capacidades intelectuales en el actual ámbito educativo.

Palabras claves: actitudes; altas capacidades; creencias; formación docente; inclusión educativa

\section{ABSTRACT}

Attitudes of teachers towards students with high intellectual capacities and the importance of teacher training in this ámbit. This research proposes to demonstrate how the specific training that teachers receive, in this case, on students with high intellectual abilities, is capable of favorably modifying previous 


\section{ACTITUDES Y CREENCIAS DE LOS DOCENTES HACIA EL ALUMNADO CON ALTAS CAPACIDADES INTELECTUALES Y LA IMPORTANCIA DE LA FORMACIÓN DEL PROFESORADO EN ESTE ÁMBITO}

beliefs and attitudes towards these students. Numerous studies consulted worldwide confirm that understanding what these students are like and what their needs are, helps teachers to improve their attitudes towards them and their educational response possibilities. In this study, using the Opinions about the gifted and their education scale, original by Gagné and Nadeau (1991), translated and adapted into Spanish, an experimental group of 167 teachers from all the educational stages, with which a quasi- experimental, pretest-posttest design was carried out.

The results indicated that indeed, after this training, some attitudes that the teachers initially presented were favorably modified, observing an improvement in terms of the subjective opinion they have of this group, towards the rejection that is initially assumed to these students and their difficulty to have good social relations and especially in the consideration of educational measures with these students, showing more predisposition towards the acceleration and flexibility of students with high capacities in the school environment, considering the educational inclusion of students with high intellectual capacities in the current environment educational field.

Keywords: attitudes; high abilities; beliefs; teacher training; educational inclusion

\section{INTRODUCCIÓN}

Observando la realidad educativa de los centros escolares en lo que al alumnado con altas capacidades (en adelante AACC) se refiere, uno de los mayores escollos el desconocimiento por parte del profesorado de las características de este alumnado en las aulas. En la base de la desatención de este alumnado, se sitúa como una de las explicaciones más evidentes la falta de formación de maestros y profesores, así como la escasez de recursos y oportunidades para este alumnado que obstaculizan su ajuste educativo, conduciéndole en muchos casos a situaciones de desmotivación y frustración ante un sistema educativo que no considera importantes sus necesidades.

Numerosos estudios corroboran como las creencias de los profesores en sus percepciones acerca del alumnado con altas capacidades, repercuten directamente en su identificación y en su respuesta a las necesidades educativas que presentan.

En este artículo, se intenta demostrar que las actitudes iniciales de un grupo de docentes extremeños, se modifican favorablemente en algunos aspectos hacia la consideración de este alumnado, tras haberse sometido a un curso formativo sobre esta temática.

\section{DEFINICIÓN DE ACTITUD}

Definir el concepto de actitud ha resultado una tarea compleja, tanto por la diversidad de elementos que incluye el propio constructo como por la inexistencia de una adecuada definición (Bautista, 2001; Del Caño, 2006). Para Arnau (2005) la actitud se define como una tendencia a actuar de una manera determinada, expresada en la evaluación positiva o negativa hacia un objeto y que está condicionada por la incidencia de los componentes cognitivos, afectivos y conductuales. Algunos autores (Azjen y Fishbein, 1980) postulan que las actitudes se adquieren automáticamente hacia un objeto, a través de asociaciones de este con otros objetos sobre los que ya se tiene una actitud previa y al mismo tiempo, se adquiriría una actitud hacia dicho objeto (Reyes, 2007). Otros autores defienden que las actitudes se pueden aprender (Trujillo, De la Poza y Portero, 2016) y que se pueden medir (Chessman, 2010) aunque no sean directamente visibles, a través de observaciones e instrumentos especiales.

Para esta investigación, se han tenido en cuenta las aportaciones de distintos autores (Ortega, 1986; Pacheco, 2002; Ubillos, Mayordomo y Páez, 2004) en las que suponen que las actitudes no son inamovibles, sí pueden aprenderse y modificarse y por tanto, desarrollarse a través de la experiencia, siendo variables mediadoras y predictivas del comportamiento (Verdugo, Arias y Jenaro, 1994) referidas a un ámbito colectivo concreto.

\section{ACTITUDES DEL PROFESORADO HACIA LA INCLUSIÓN EDUCATIVA}

La inclusión, supone que tanto el sistema educativo como el currículo, deben adaptarse a las características y necesidades educativas del alumnado y no al contrario. Para ello, es imprescindible convertir las escuelas en 
centros que respondan a toda la diversidad del alumnado (Ainscow, 2012). Así, un modelo educativo que se base en la equidad, la participación y la efectividad (Garzón, Calvo y Orgaz, 2016) debe tener como objetivos desarrollar las capacidades del alumnado, adaptarse a la diversidad de sus necesidades, independientemente de cuáles sean sus condiciones personales, sociales o culturales, intentando cambiar modelos educativos tradicionales por otros más flexibles que respondan a todos alumnos (Escarbajal, 2010; 2012).

Desde estos planteamientos, el docente se convierte en el principal instrumento educativo en la construcción de una escuela inclusiva de calidad (Jordán, 2007; Palomares, 2011) y aunque la mayoría de los profesores apoya la inclusión, su convencimiento disminuye cuando deben implicarse en la atención a esa diversidad (Pelajar y Colmenero, 2017).

\section{ACTITUDES DEL PROFESORADO HACIA LA ALTA CAPACIDAD}

Si la inclusión educativa en materia de atención a la diversidad, supone un reto para los docentes, en el caso de los alumnos con necesidades específicas de apoyo educativo por tener altas capacidades intelectuales, es aún mayor. Las actuaciones educativas distintas con este alumnado, en el marco de una educación inclusiva, sigue siendo un asunto dependiente de las actitudes que muestren los docentes y no sólo de recursos materiales y organizativos, técnicas metodológicas o cambios en el currículo de este alumnado (González y Palomares, 2016; Hernández y Gutiérrez, 2014).

Las creencias, incluso las erróneas, que tiene un docente sobre las capacidades o posibilidades de un alumno, predispone el comportamiento de ese alumno, favorable o desfavorablemente (Pomar, 2011) y conducen a evaluaciones negativas de estos alumnos y a un sesgo en su identificación, sobre todo si muestran dificultades para ajustarse al entorno educativo (Preckel, Baudson, Krolack-Schwerdt y Glock,2015).

Por tanto, para que un alumno con AACC exprese su capacidad en el entorno escolar, es imprescindible que el profesorado manifieste actitudes positivas hacia este campo (Sánchez, 1997) y conocer las características de este alumnado (Del Caño, 2001). De hecho, el conocimiento de estos alumnos y la actitud hacia ellos, está relacionados (Genzel y Satmaz, 2017; Plunkett, 2000) de ahí, la importancia de la formación específica en AACC para fomentar actitudes favorables en el profesorado.

Resulta pues fundamental, que los docentes desarrollen actitudes apropiadas hacia los estudiantes con AACC basados en creencias cognitivas precisas, ya que si no es así, sus sentimientos y comportamientos hacia esta población, pueden estar sesgados (Toxclair, 2013).

\section{OBJETIVOS DE LA INVESTIGACIÓN}

El objetivo general de este estudio pretende explorar las actitudes de los docentes hacia las AACC y cómo influye la formación específica que reciban en éste ámbito, en el cambio favorable de sus actitudes hacia este alumnado.

De forma más específica, esta investigación pretende probar los efectos de una intervención sobre las actitudes del profesorado participante, a partir de un programa formativo, en el que se imparten contenidos relacionados con las características y necesidades educativas de estos alumnos.

\section{MUESTRA Y/O PARTICIPANTES}

En este estudio, se optó por una metodología de tipo cuasi-experimental, con un diseño pretest -postest con grupo control. Se han recopilado los datos obtenidos por los docentes que asisten a los cursos de formación específica en AACC, con un total de 167 participantes, mayoritariamente de las etapas de Educación Primaria y Secundaria. De ellos, 127 lo hacen en los Centros de Profesores y Recursos, CPRs (GE1), siendo 92 mujeres y 35 docentes varones. El resto de participantes, 40 (GE2), 26 mujeres y 14 varones, lo hace en su centro educativo concertado. Se invalidan previamente dos cuestionarios del GE1. El Grupo Control (GC), se compone de 19 


\section{ACTITUDES Y CREENCIAS DE LOS DOCENTES HACIA EL ALUMNADO CON ALTAS CAPACIDADES INTELECTUALES Y LA IMPORTANCIA DE LA FORMACIÓN DEL PROFESORADO EN ESTE ÁMBITO}

docentes, 4 de ellos varones y 15 mujeres, participantes de un curso del CPR ajeno a la temática de las AACC y varios docentes de un Centro Concertado de otra ciudad.

La media de edad es de 41.5 años $(D T=9.08$ ) en el GE1 (los asistentes al CPR), Ios del GE2 (centro educativo), 46.8 años ( $D T=9.71$ ) y los del $G C$, una media de 31.8 años $(D T=9.36)$. Los años de experiencia docente, oscilan entre una media de 14 años ( $D T=.95)$ para los asistentes a CPRs y $21.32(D T=11)$ años para los del centro educativo.

Prácticamente la mitad de los participantes, declara no haber recibido nunca formación sobre este alumnado y valoran bajo o muy bajo su nivel formativo en la atención a los alumnos con AACC, aunque la mayoría afirma conocer a algún alumno con AACC. Entre los docentes en este estudio, se observa que la media de alumnos con AACC identificados por los participantes, es discreta $(M=1.12, D T=2.55)$ para los docentes que acuden a la formación en los CPRs (GE1), siendo análoga en el grupo de participantes del centro escolar (GE2), donde un 52.5\% declara que nunca ha identificado a ningún alumno con AACC en su práctica docente.

\section{METODOLOGÍA Y/O INSTRUMENTOS UTILIZADOS}

En el primer contexto (GE1), se solicitó la participación voluntaria de maestros y profesores que acudieron a un curso de formación, en lugares oficiales destinados para ello (Centro de Profesores y Recursos, con una duración de 15 horas) yen el segundo, se realizó la instrucción, con docentes de un colegio concertado (con una duración de 9 horas). El programa de contenidos sobre altas capacidades intelectuales fue el mismo en todos los cursos impartidos. El desarrollo de esta intervención, como indican la siguiente figura, se vinculaba a las siguientes fases:

Figura 1. Fases de la intervención..

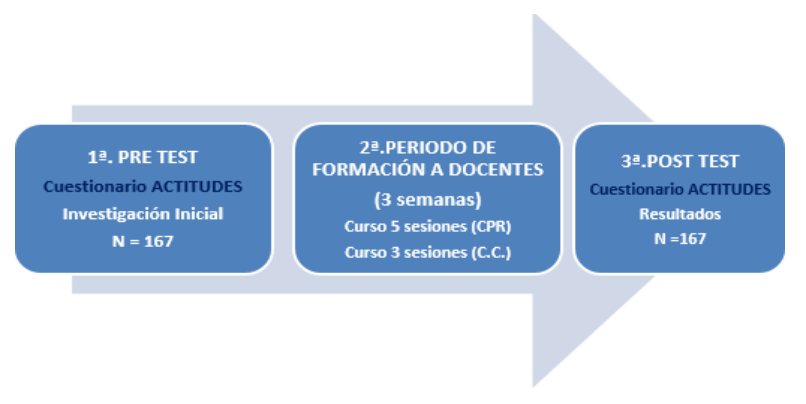

Las actitudes de los docentes hacia los estudiantes con AACC y su educación en Extremadura, se obtuvieron utilizando la escala original de Gagné y Nadeau "Opinions about the gifted and their education", ya que ha resultado ser tras una amplica revisión bibliográfica, la más empleada en el ámbito internacional (Al Garni, 2012; Allodi y Rydelius, 2008; Gencel y Satman, 2017; Lassig, 2003, McCoach y Seigle, 2007; Seade, Encalada, Peñaherrera, Dávila y Vélez, 2019; Toxclair, 2013; Watts, 2006) para evaluar actitudes. Ha sido traducida y validada al español por Tourón y Reyero (2002) y utilizada en nuestro país por Vilaseca (2004) y Roa (2017) en adaptaciones a las poblaciones catalana y vasca, respectivamente. La escala, se ha adaptado teniendo en cuenta la normativa y nomenclatura con la que designamos términos docentes relativos a las AACC en Extremadura. Consta de 34 ítems distribuidos en seis factores (Necesidades Educativas; Opiniones Subjetivas; Reconocimiento Social; Rechazo; Medidas

Educativas; Medidas Educativas Excepcionales) evaluados con una escala sumativa de Likert, con opciones de respuesta: 1. Totalmente en desacuerdo; 2. Parcialmente en desacuerdo; 3. Indeciso; 4. Parcialmente de acuerdo; 5 . Totalmente de acuerdo. Medias por debajo de 2.0 (por encima de 4.00 en el factor 2) normalmente indica 
una actitud muy negativa; la media sobre 4.00 tiene el significado opuesto 0 contrario, es decir actitud muy positiva; medias entre 2 y 2.75 , indicaría una actitud ligeramente negativa; media entre 2.75 y 3.25 se podría interpretar como el reflejo de una actitud ambivalente y la media entre 3.25 y 4 , indicaría actitudes ligeramente positivas.

\section{RESULTADOS ALCANZADOS}

Con la intención de comprobar si la formación en AACC influye positivamente en las actitudes de los participantes, se realizan comparaciones de medias intergrupos (pretets-postest) que nos indican que la intervención desarrollada con los participantes, (tanto en el GE1 como en GE2), ha resultado eficaz, en comparación con los resultados obtenidos por los docentes participantes en el GC. En el GE1 se observan actitudes más favorables en todos los factores, Opinión subjetivas (OS), Rechazo (RE), Medidas Educativas (ME) y Medidas Educativas Excepcionales (MEE). a excepción del factor Reconocimiento Social (RS), mientras que, en el GE2, sólo se observaron cambios significativos en las dimensiones OS y MEE. En cuanto a las puntuaciones obtenidas por los participantes en el Grupo Control (GC), únicamente encontramos diferencias significativas en la variable ME.

Siguiendo estudios previos (AI Garni, 2012; Toxclair, 2013), realizamos un análisis pormenorizado de los ítems, en los distintos factores en los que hemos observado cambios. Mostramos el porcentaje de los resultados y calculamos las medias de los ítems, no para compararlas entre sí, sino para situar en qué categoría estarían las actitudes evaluadas, muy negativa, ligeramente negativa, ambivalente, ligeramente positiva o muy positiva (Gagné, 1991).

\section{Cambios significativos en actitudes en el factor Opinión Subjetiva en los GE1 y GE}

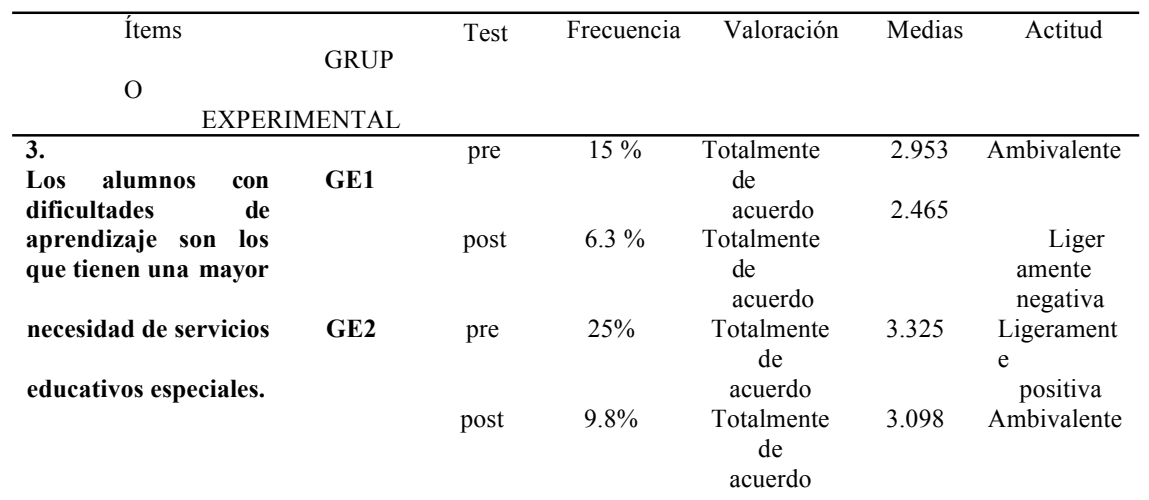

Antes de la formación, observamos más profesores de acuerdo con esta afirmación, presentando actitudes más ambivalentes que modifican tras el curso formativo con más horas de duración, mostrando más descuerdo hacia la consideración de que los alumnos con dificultades de aprendizaje tengan una atención educativa específica prioritaria a los de AACC. Ocurre igual que en GE2, aunque las actitudes son más neutras hacia esta consideración. 


\begin{tabular}{|c|c|c|c|c|c|c|}
\hline $\begin{array}{l}4 . \\
\text { Los programas }\end{array}$ & GE1 & pre & $29.4 \%$ & $\begin{array}{l}\text { Totalmente } \\
\text { en } \\
\text { desacuerdo }\end{array}$ & 2.468 & $\begin{array}{c}\text { Ligerament } \\
\mathrm{e} \\
\text { positiva }\end{array}$ \\
\hline $\begin{array}{l}\text { especiales } \\
\text { alumnos con altas } \\
\text { capacidades tienen la } \\
\text { desventaja de crear }\end{array}$ & & post & $49.2 \%$ & $\begin{array}{l}\text { Totalmente } \\
\text { en } \\
\text { desacuerdo }\end{array}$ & 1.811 & Muy negativa \\
\hline & GE2 & pre & $40 \%$ & Totalmente & & \\
\hline & en & post & $585 \%$ & $\begin{array}{l}\text { desacuerdo } \\
\text { Totalmente } \\
\text { en } \\
\text { desacuerdo }\end{array}$ & 2.415 & $\begin{array}{l}\text { Liger } \\
\text { amente } \\
\text { negativa }\end{array}$ \\
\hline
\end{tabular}

Tras la formación más amplia en horas, se duplica el porcentaje de docentes que no están de acuerdo con que los programas especiales para AACC creen elitismo, modificando una actitud previa ligeramente positiva hacia esto, a estar muy en contra de ella tras la formación.Ocurre igual que en GE2. Más docentes en desacuerdo con esta afirmación, aunque el efecto es menor que en el GE1.

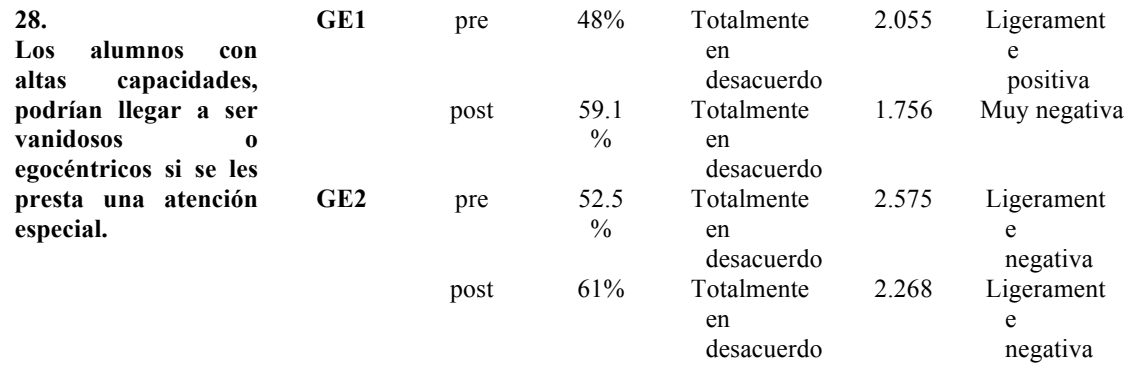

Tras la formación, aumenta el porcentaje de profesores que no está de acuerdo con que los alumnos por recibir una atención educativa específica se muestran engreídos. Aumenta la actitud negativa hacia este ítem.

\section{Cambios significativos en actitudes en el factor Rechazo en los GE1 y GE2}

\begin{tabular}{|c|c|c|c|c|c|c|}
\hline Ítems & $\begin{array}{l}\text { GRUP } \\
\text { O } \\
\text { EXPERI } \\
\text { MENTA } \\
\text { L }\end{array}$ & $\begin{array}{c}\text { Tes } \\
t\end{array}$ & $\begin{array}{c}\text { Frecue } \\
\text { ncia }\end{array}$ & $\begin{array}{l}\text { Valoraci } \\
\text { ón }\end{array}$ & $\begin{array}{l}\text { Media } \\
\mathrm{s}\end{array}$ & Actitud \\
\hline \multirow[t]{3}{*}{$\begin{array}{l}19 . \\
\text { Un alumno que ha } \\
\text { sido identificado de } \\
\text { altas capacidades, } \\
\text { tiene más } \\
\text { dificultades para } \\
\text { hacer amigos. }\end{array}$} & GE1 & post & $50.4 \%$ & $\begin{array}{l}\text { Totalmente } \\
\text { en } \\
\text { Desacuerdo } \\
\text { Totalmente } \\
\text { en } \\
\text { Desacuerdo }\end{array}$ & 2.055 & $\begin{array}{l}\text { Ligerament } \\
\mathrm{e} \\
\text { Negativa } \\
\text { Ligerament } \\
\mathrm{e} \\
\text { Negativa }\end{array}$ \\
\hline & GE2 & pre & $50 \%$ & $\begin{array}{l}\text { Totalmente } \\
\text { en } \\
\text { Desacuerdo }\end{array}$ & 2.725 & $\begin{array}{c}\text { Ambivalent } \\
\mathrm{e}\end{array}$ \\
\hline & & post & $63.4 \%$ & $\begin{array}{l}\text { Totalmente } \\
\text { en } \\
\text { Desacuerdo }\end{array}$ & 2.317 & $\begin{array}{l}\text { Ligerament } \\
\mathrm{e} \\
\text { Negativa }\end{array}$ \\
\hline
\end{tabular}


En este ítem, resultan más evidentes los cambios producidos en el periodo formativo de 9h, siendo más numerosos los docentes que tras la instrucción no cree que los alumnos con AACC tengan más dificultad para hacer amigos.

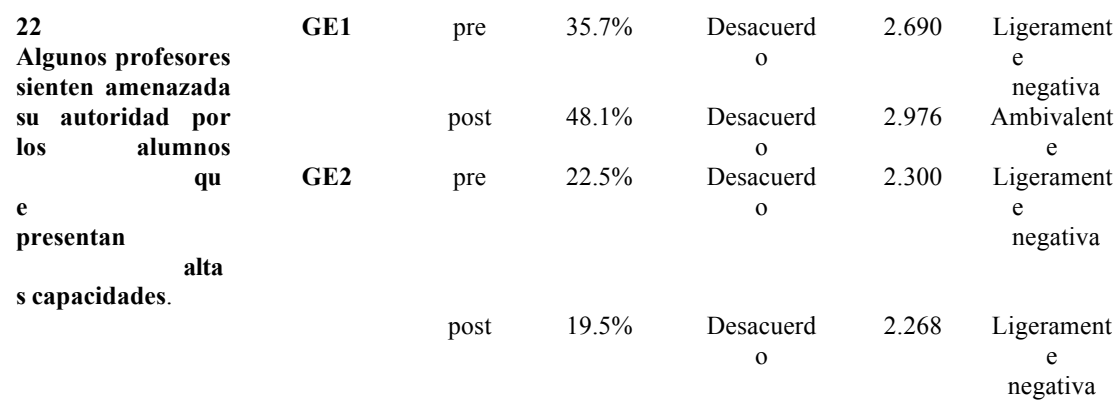

Tras la formación de más horas, se observa un cambio favorable de actitud de los participantes en el hecho de ver cuestionada su autoridad por estos alumnos. No lo perciben tan negativamente tras esta formación.En el grupo de docentes con menos horas, no se modifica esta actitud tras la instrucción.

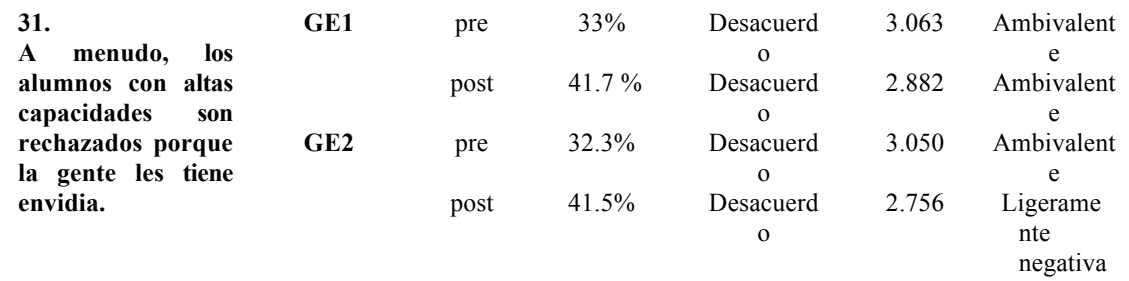

En el GE1, no se observan cambios, aunque son más los docentes que están en desacuerdo con este ítem. Se observan actitudes más positivas en los docentes del GE2 respecto al rechazo que suponen a estos alumnos por los demás tras la formación.

\section{Cambios significativos en actitudes en el factor Medidas Educativas en los GE1 y GE2}

\begin{tabular}{|c|c|c|c|c|c|c|}
\hline Ítems & $\begin{array}{l}\text { GRUP } \\
\text { O } \\
\text { EXPERIM } \\
\text { ENTAL } \\
\end{array}$ & Test & $\begin{array}{l}\text { Frecuenc } \\
\text { ia }\end{array}$ & Valoración & Medias & Actitud \\
\hline $\begin{array}{l}6 . \\
\text { Cuando se sitúa a }\end{array}$ & GE1 & pre & $29.1 \%$ & $\begin{array}{l}\text { Totalmente } \\
\text { en } \\
\text { desacuerdo }\end{array}$ & 2.354 & $\begin{array}{c}\text { Ligerament } \\
\text { e } \\
\text { Negativa }\end{array}$ \\
\hline $\begin{array}{l}\text { los alumnos de } \\
\text { altas capacidades }\end{array}$ & & post & $48,8 \%$ & $\begin{array}{l}\text { Totalmente } \\
\text { en } \\
\text { desacuerdo }\end{array}$ & 1.906 & $\begin{array}{c}\text { Muy } \\
\text { Negativa }\end{array}$ \\
\hline en clases & & pre & $15 \%$ & $\begin{array}{c}\text { Totalmente } \\
\text { en }\end{array}$ & 2.775 & $\begin{array}{c}\text { Ambivalent } \\
\mathrm{e}\end{array}$ \\
\hline $\begin{array}{l}\text { especiales, los } \\
\text { otros }\end{array}$ & GE2 & & & desacuerdo & & \\
\hline $\begin{array}{l}\text { se sienten } \\
\text { infravalorados. }\end{array}$ & & post & $17 \%$ & $\begin{array}{c}\text { Totalmente } \\
\text { en } \\
\text { desacuerdo }\end{array}$ & 2.756 & $\begin{array}{c}\text { Ambivalent } \\
\mathrm{e}\end{array}$ \\
\hline
\end{tabular}


Tras la formación más extensa, se observan cambios en las actitudes de los docentes, ya que se incrementa evidentemente el porcentaje de ellos que no está de acuerdo con esta afirmación. Son, por tanto, más reacios a pensar que situar a los niños con AACC en otra clase, sea un perjuicio para el resto de alumnos. No se modifican las actitudes en el GE2 sometido a menos horas formativas en cuanto a este ítem

\begin{tabular}{|c|c|c|c|c|c|c|}
\hline 20. & GE1 & pre & $11 \%$ & Totalmente en & 3.189 & Ambivalente \\
\hline $\begin{array}{l}\text { Los alumnos con } \\
\text { altas capacidades } \\
\text { deberían }\end{array}$ & & post & $24.4 \%$ & $\begin{array}{l}\text { desacuerdo } \\
\text { Totalmente en } \\
\text { desacuerdo }\end{array}$ & 2.559 & $\begin{array}{l}\text { Ligeramente } \\
\text { negativa }\end{array}$ \\
\hline $\begin{array}{l}\text { permanecer en las } \\
\text { clases }\end{array}$ & GE2 & pre & $7.5 \%$ & $\begin{array}{l}\text { Tgtalmente en } \\
\text { desacuerdo }\end{array}$ & 3.250 & $\begin{array}{l}\text { Ligeramente } \\
\text { positiva }\end{array}$ \\
\hline $\begin{array}{l}\text { regulare } \\
\text { s, puesto que son } \\
\text { un estímulo } \\
\text { intelectual } \\
\text { para }\end{array}$ & & post & $7.3 \%$ & $\begin{array}{l}\text { Totalmente } \\
\text { en } \\
\text { desacuerdo }\end{array}$ & 3.000 & Ambivalente \\
\hline
\end{tabular}

Tras la formación, cambia favorablemente la actitud de los profesores hacia la consideración de opciones educativas distintas con estos alumnos, en ambos grupos experimentales, más evidentemente en el GE1 que en el GE2, que se muestran menos de acuerdo, con que permanezcan en la misma clase que sus iguales sin que sea en su beneficio.

\begin{tabular}{|c|c|c|c|c|c|c|}
\hline $\begin{array}{lr}21 . & \\
\text { Separando } & \text { los } \\
\text { estudiantes } & \text { en }\end{array}$ & GE1 & pre & $22 \%$ & $\begin{array}{l}\text { Totalmente } \\
\quad \text { de } \\
\text { acuerdo } \\
\text { Totalmente de }\end{array}$ & 3.370 & $\begin{array}{l}\text { Ligerament } \\
\mathrm{e} \\
\text { Positiva } \\
\text { Ambivalent } \\
\mathrm{e}\end{array}$ \\
\hline $\begin{array}{l}\text { alumnos con altas } \\
\text { capacidades } y \\
\text { otros }\end{array}$ & GE2 & pre & $32.5 \%$ & $\begin{array}{c}\text { Acuerdo } \\
\text { Totalmente } \\
\text { de acuerdo }\end{array}$ & 3.650 & $\begin{array}{c}\text { Ligerament } \\
\text { e Positiva }\end{array}$ \\
\hline \begin{tabular}{l}
\multicolumn{2}{c}{ grupos } \\
, estamos \\
incrementando el \\
etiquetaje de los \\
mismos.
\end{tabular} & & post & $14.6 \%$ & $\begin{array}{c}\text { Totalmente } \\
\text { de } \\
\text { acuerdo }\end{array}$ & 3.195 & $\begin{array}{c}\text { Ambivalen } \\
\text { te }\end{array}$ \\
\hline
\end{tabular}

Se observa un cambio en la actitud de los docentes, hacia medidas de agrupamiento diferentes para el alumnado de AACC, tras la formación en los dos periodos formativos, ya que en ambos casos el porcentaje de docentes que están de acuerdo con esta afirmación disminuye claramente. Se muestran más indecisos a pensar que separarlos por capacidad sea etiquetarlos.

\section{Cambios significativos en actitudes en el factor Medidas Educativas Excepcionales en Ios GE1 y GE2}




\begin{tabular}{|c|c|c|c|c|c|c|}
\hline Ítems & \begin{tabular}{l}
\multicolumn{1}{c}{ GRUPO } \\
EXPERIMENT \\
AL
\end{tabular} & Test & $\begin{array}{l}\text { Frecuenc } \\
\text { ia }\end{array}$ & Valoración & $\begin{array}{c}\text { Media } \\
\mathrm{s}\end{array}$ & Actitud \\
\hline $\begin{array}{l}7 . \\
\text { La mayoría de los } \\
\text { alumnos con altas }\end{array}$ & GE1 & pre & $11.1 \%$ & $\begin{array}{l}\text { Totalmente } \\
\text { en } \\
\text { Desacuerdo }\end{array}$ & 2.944 & $\begin{array}{c}\text { Ambivalent } \\
\mathrm{e}\end{array}$ \\
\hline $\begin{array}{l}\text { capacidades } \\
\text { qu } \\
\text { e avanzan un curso }\end{array}$ & & post & $38.9 \%$ & $\begin{array}{l}\text { Totalmente } \\
\text { en } \\
\text { Desacuerdo }\end{array}$ & 2.103 & $\begin{array}{l}\text { Ligerament } \\
\mathrm{e} \\
\text { negativa }\end{array}$ \\
\hline $\begin{array}{l}\text { tienen dificultades } \\
\text { para relacionarse } \\
\text { socialmente con el }\end{array}$ & GE2 & pre & $12.8 \%$ & $\begin{array}{l}\text { Totalmente } \\
\text { en } \\
\text { Desacuerdo }\end{array}$ & 2.949 & $\begin{array}{c}\text { Ambivalent } \\
\mathrm{e}\end{array}$ \\
\hline $\begin{array}{ll}\text { grupo } & \text { de } \\
\text { estudiantes } \\
\text { mayores }\end{array}$ & & post & $10 \%$ & $\begin{array}{l}\text { Totalmente } \\
\text { en } \\
\text { Desacuerdo }\end{array}$ & 2.650 & $\begin{array}{l}\text { Ligerament } \\
\mathrm{e} \\
\text { negativa }\end{array}$ \\
\hline
\end{tabular}

Tras la formación, los docentes muestran una actitud más favorable en la consideración de que avanzar un curso en los alumnos con AACC, no supone dificultades para relacionarse socialmente con compañeros más mayores. El porcentaje de los docentes que no está de acuerdo con la afirmación del ítem se incrementa notablemente tras recibir el curso. Aunque se observan cambios en las actitudes, el porcentaje de docentes que están en total desacuerdo con_esta afirmación es menor en el GE2.

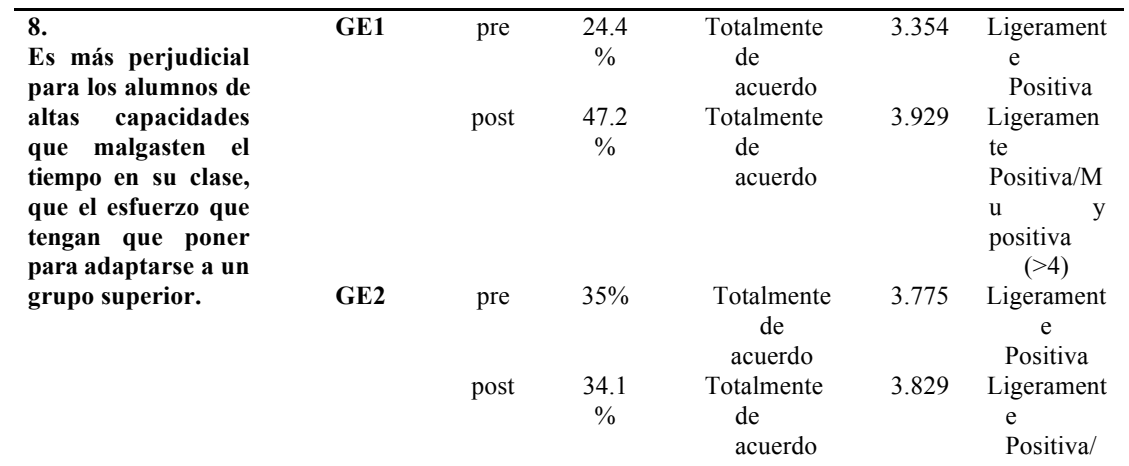

Tras la formación, se observan cambios en las actitudes de los docentes, ya que casi el doble de ellos, muestra actitudes muy favorables ante la aceleración/flexibilización de estos alumnos, mostrando tras la realización del curso con más horas formativas, mejor actitud hacia medidas excepcionales que en el caso del GE2 con menos horas deformación.

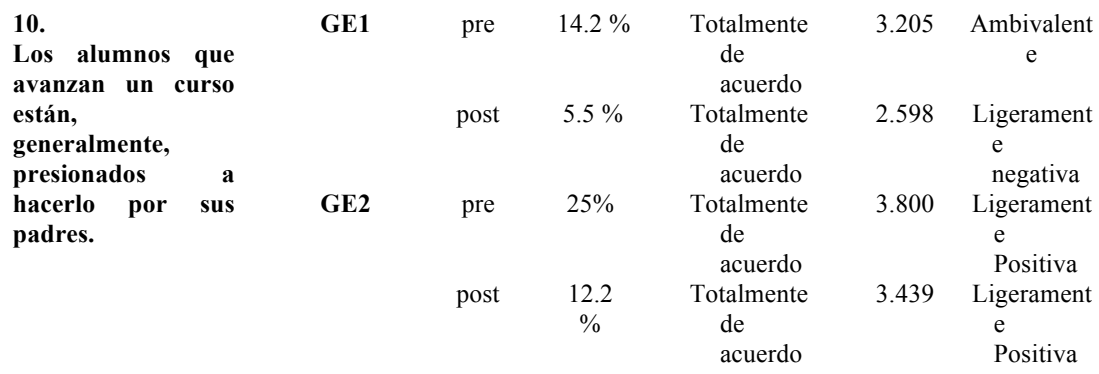




\section{ACTITUDES Y CREENCIAS DE LOS DOCENTES HACIA EL ALUMNADO CON ALTAS CAPACIDADES INTELECTUALES Y LA IMPORTANCIA DE LA FORMACIÓN DEL PROFESORADO EN ESTE ÁMBITO}

Tras la formación de $15 \mathrm{~h}$, cambia la actitud y la percepción inicial de los docentes ante su creencia de que son los padres quienes presionan a sus hijos ante las medidas excepcionales de aceleración o flexibilización. No se observan cambios en la actitud de los docentes sometidos a menos horas de formación, si bien es menor el porcentaje de ellos que está de acuerdo con esta afirmación.

\begin{tabular}{|c|c|c|c|c|c|c|}
\hline \multirow{5}{*}{$\begin{array}{l}29 . \\
\text { Cuando los } \\
\text { alumnos con altas } \\
\text { capacidades } \\
\text { avanzan un curso, } \\
\text { corren el riesgo de } \\
\text { tener "lagunas" en } \\
\text { su conocimiento. }\end{array}$} & \multirow{4}{*}{$\begin{array}{l}\text { GE1 } \\
\text { GE2 }\end{array}$} & pre & $32.3 \%$ & & 2.205 & \multirow{3}{*}{$\begin{array}{l}\text { Ligerament } \\
\text { e } \\
\text { Negativa } \\
\quad \text { Muy } \\
\text { Negativ } \\
\text { a }\end{array}$} \\
\hline & & & & Desacuerdo & & \\
\hline & & post & $\begin{array}{c}49.6 \\
\%\end{array}$ & & 1.992 & \\
\hline & & pre & $5 \%$ & $\begin{array}{l}\text { Desacuerdo } \\
\text { Totalmente } \\
\text { en }\end{array}$ & 2.375 & $\begin{array}{l}\mathrm{a} \\
\text { Ligerament } \\
\mathrm{e}\end{array}$ \\
\hline & & post & $7.3 \%$ & $\begin{array}{l}\text { Desacuerdo } \\
\text { Totalmente } \\
\text { en } \\
\text { Desacuerdo }\end{array}$ & 2.415 & $\begin{array}{c}\text { Negativa } \\
\text { Ligerament } \\
\mathrm{e} \\
\text { Negativa }\end{array}$ \\
\hline
\end{tabular}

Aunque un tercio de los participantes ya mostraban su desacuerdo y una actitud ligeramente negativa hacia esta afirmación, tras el periodo formativo con más carga horaria, aumenta la actitud de los docentes, considerando casi la mitad de ellos tras la formación que no suponen lagunas de conocimiento en los alumnos que aceleran o flexibilizan un curso. En los docentes con menos horas de formación, no se observan modificaciones en la actitud en este ítem.

\begin{tabular}{|c|c|c|c|c|c|c|}
\hline \multirow{3}{*}{$\begin{array}{l}34 . \\
\text { Se debería permitir } \\
\text { avanzar un curso a } \\
\text { un mayor número } \\
\text { de } \\
\text { alumnos con altas } \\
\text { capacidades. }\end{array}$} & GE1 & pre & $\begin{array}{c}24.4 \\
\%\end{array}$ & De acuerdo & 3.354 & $\begin{array}{l}\text { Ligerame } \\
\text { nte }\end{array}$ \\
\hline & & post & $\begin{array}{c}44,9 \\
\%\end{array}$ & De acuerdo & 4.118 & $\begin{array}{c}\text { Positiva } \\
\text { Muy } \\
\text { positiva }\end{array}$ \\
\hline & GE2 & pre & 32.5 & De acuerdo & 3.200 & $\begin{array}{c}\text { Ambivalent } \\
\mathrm{e}\end{array}$ \\
\hline
\end{tabular}

Tras la formación más extensa, se evidencia un aumento importante de docentes que muestra actitudes favorables hacia la flexibilización como recurso más generalizado de atención educativa a los alumnos con AACC. Ocurre lo mismo en el grupo que menos horas formativas recibe, si bien, el cambio de actitudes es más favorable en el GE1.

\section{DISCUSIÓN}

Se ha comprobado la eficacia de la formación específica en el cambio favorable de las actitudes de los docentes participantes en las necesidades educativas del alumnado con AACC. Estudios previos internacionales, coinciden con esta investigación, concluyendo que mejorar la comprensión sobre las características de los AACC, se asocia a mejoras significativas en las actitudes de los futuros docentes (Al Garni, 2012; Bangel,

Enersen, Capobianco y Moon, 2006; Gross, 1997; Heckenberg 2001; Plunkett, 2000, Hudson, P., Hudson, S., Lewis y Watters, 2010; Morrissey, 2006). Estudios nacionales, coinciden en afirmar la misma relación (Del Caño, 2001; García-Barrera y De la Flor, 2016; Mendioroz, Rivero y Aguilera, 2019; González, Palomares y Domingo, 2017; Peña, 2016; Tourón, Fernández, Reyero, 2002). Otros estudios no muestran una clara relación entre la participación en cursos formativos y el cambio de actitudes (Begin y Gagné, 1994).

En este estudio, han resultado más llamativos los cambios registrados en el factor Opinión Subjetiva tras la instrucción, mejorando aquellas actitudes referidas a la diferenciación educativa de este alumnado, coincidiendo 
con estudios previos como el de Tourón, Fernández y Reyero (2002). Sin embargo, en otros estudios (McCoach y Siegle, 2007; Seade et al., 2019 y Toxclair, 2013) no se observan diferencias significativas en este factor (OS) tras la formación específica de los profesores. En cuanto al factor Rechazo, se han observado cambios respecto a las actitudes iniciales hacia estos alumnos, al igual que en los estudios de Al Garni (2012) y Allodi y Rydelius (2008). Respecto ala consideración de Medidas Educativas ordinarias en nuestro sistema (agrupación de habilidades) se observaron cambios favorables tras el periodo formativo de los docentes, sobre todo en el GE1, coincidiendo con Watts (2006) y Polyzopoulos, Kokaridas, Patsiaosauras y Gari (2014). Modificaciones más significativas en las actitudes favorables de los docentes en la consideración hacia las Medidas Educativas Excepcionales (aceleración/flexibilización), coincidiendo así con otros estudios previos (Al Garni, 2012; Polyzopoulos et al.,2014; Tourón et al., 2002; Seade et al., 2019).

\section{CONCLUSIONES}

En esta investigación, se modifican favorablemente las creencias previas que tenían los docentes hacia el alumnado con AACC y sus características, tras la formación, y esto repercutirá sustancialmente en la interacción con los alumnos en el contexto escolar (Voss et al., 2013). Es un cambio muy positivo, ya que la formación en cómo son estos alumnos, cambia la actitud de muchos docentes, desestimando la relación atención educativa y elitismo. Igualmente, observamos que aumenta el número de participantes que piensan que tener AACC, no influye en su comportamiento diferente o petulante, e incluso más profesores reconocen que deben atenderse educativamente, igual que cualquier otro alumno con dificultades de aprendizaje.

Por tanto, es fundamental una formación específica y de calidad en los docentes, que acerque a la realidad educativa de los centros a los alumnos con AACC y fomentar creencias positivas en el profesorado hacia ellos, ya que es la única vía para tomar decisiones acertadas (Barrenetxea y Martínez, 2019; Tourón, Fernández y Reyero, 2002). Las aulas, son cada vez más diversas, es cierto, y en el caso de los alumnos con AACC también se da esa diversidad, siendo el papel del profesorado, crucial y decisivo en el conocimiento de estos alumnos, en su adaptación positiva al entorno escolar y a su proceso de aprendizaje. Esto significa ser escuela inclusiva y atender a la diversidad, también a los alumnos de AACC.

\section{REFERENCIAS BIBLIOGRÁFICAS}

Ainscow, M., Dyson, A., Goldrick, S., y West, M. (2012). Making schools effective for all: rethinking the task. School Leadership \& Management, 32(3), 197-213.

Aizen, I. y Fishbein, M. (1980). Determinants of behavioral intentions. Understanding attitudes and predicting social behavior. Englewood Cliffs (NJ): Prentice-Hall, 53-60.

Al Garni, A.A. (2012). Attitudes of Future Special Education Teachers Toward Gifted Students and their Education. Doctoral dissertation. Faculty of Education Queensland University of Technology.

Allodi, M. W., y Rydelius, P. (septiembre2008). Gifted children their school environments, mental health and specific needs: A study of Swedish teachers' knowledge and attitudes. Paper presented at the European Council for High Ability Conference, Prague, Czech Republic.

Arnau, A. y Montané, J. M. (2017). Aportaciones sobre la relación conceptual entre actitud y competencia, desde la teoría del cambio de actitudes. Electronic Journal of Research in Education Psychology, 8 (22), 1283-1302.

Barrenetxea, L., y Martínez, M. (2019). Relevancia de la formación docente para la inclusión educativa del alumnado con altas capacidades intelectuales. Atenas, 1 (49), 1-19.

Bautista, J. M. (2001). Actitudes y valores: precisiones Conceptuales para el trabajo. XXI, Revista de Educación, 3, 189-196.

Bangel, N.J, Enersen, D., Capobianco, B. y Moon, S.M, (2006). Desarrollo profesional de docentes en servicio: enseñanza en el programa súper sábado. Revista para la Educación de los Dotados, 29 (3), 339-361

Begin, J. y Gagné, F. (1994). Predictor of a general attitude toward gifted education. Journal for the Education of the Gifted, 18 (1), 74-86. 


\section{ACTITUDES Y CREENCIAS DE LOS DOCENTES HACIA EL ALUMNADO CON ALTAS CAPACIDADES INTELECTUALES Y LA IMPORTANCIA DE LA FORMACIÓN DEL PROFESORADO EN ESTE ÁMBITO}

Chessman, A. M. (2010). Teacher attitudes and effective teaching practices for gifted Students at stage 6. (Unpublished doctoral tesis). The University of New South Wales, Sydney, Australia.

Del Caño Sánchez, M. (2001). Formación Inicial del Profesorado y atención a la diversidad: alumnos superdotados. Revista Interuniversitaria de Formación del Profesorado, 40,135-146.

Del Caño, M., Arias, B., Alonso, J., Morentin, R. y Gómez, H. (2006). Actitudes hacia la superdotación de aspirantes a profesores y profesores en ejercicio. En F. Cacáicoa y J.D. Uriarte: Psicología del aprendizaje. (pp.159172). Badajoz: Psicoex.

Escarbajal, A. (2010). La escuela inclusiva en una sociedad pluricultural y la importancia del trabajo colaborativo. Enseñanza y Teaching, 28(2), 161-179.

Escarbajal, A., Leiva, J. y López, J.I. (2012). La Educación Inclusiva en la escuela. En Miralles, P., y Mirete, A. La formación del profesorado en Educación Infantil y Educación Primaria, (pp. 29-36). Universidad de Murcia.

Gagné, F. y Nadeau, L. (1991): Brief Presentation of Gagné and Nadeau s Attitud Scale :Opinions about the Gifted and Their Education, Montréal. GIREDT Center, Université du Québec á Montréal.

García-Barrera, A. y de la Flor, P. (2016). Percepción del profesorado español sobre el alumnado con altas capacidades. Estudios pedagógicos (Valdivia), 42(2), 129- 149.

Garzón, P., Calvo, M.I. y Orgaz, B. (2016): Inclusión educativa. Actitudes y estrategias del profesorado. Revista Española de Discapacidad, 4 (2): 25-45. doi:10.5569/2340-5104.04.02.02.

Gencel, . E. y Satmaz, . (2017). Teacher candidates' attitudes towards education of gifted Students. UluslararasI E itim Programları ve Ö retim Çalı maları Dergisi, 7(14), 49-62.

González, R., Palomares, A. y Domingo. B, (2017). Factores desencadenantes de la actitud del profesorado hacia la inclusión educativa. En A. Rodríguez-Martín, (Comp). Prácticas Innovadoras inclusivas: retos y oportunidades. (pp.365-372). Oviedo: Universidad de Oviedo.

Gross, M. U. (1997). Changing teacher attitudes towards gifted children: An early and essential step. In J. Chan, R. Li y J. Spinks (Eds.), Maximising potential: Lengthening and strengthening our stride (pp. 3-22). Hong Kong: University of Hong Kong Social Sciences Research Centre.

Hernández, D. y Gutiérrez, M. (2014). El estudio de la alta capacidad intelectual en España: análisis de la situación actual. Revista de Educación, 364, 251-272.

Heckenberg, L. (2001). Teacher attitude toward gifted learners and gifted education: An analysis of the effectiveness of the Connie Belin Teacher Training program 1981 - 1996. In N. Colangelo y S. G. Assouline (Eds.), Talent development: Proceedings of the 1998 Henry B. and Jocelyn Wallace national research symposium on talent development (pp. 351-355). Scottsdale, AZ: Great Potential Press.

Hudson, P., Hudson, S., Lewis, K. y Watters, J. J. (2010). Embedding gifted education in preservice teacher education: A collaborative school-university approach. Australasian Journal of Gifted Education, 19 (2), 515

Jordán, J. A. (2007). Educar en la convivencia en contextos multiculturales. En E. Soriano (Coord.), Educación para la convivencia intercultural (pp. 59-94). Madrid: La Muralla.

Lassig, C. J. (2003). Teachers' attitudes towards intellectually gifted children and their education. (Masters Tesis). Griffith University, Queensland, Australia. http://eprints.qut.edu.au/14473/

McCoach, D. B. y Siegle, D. (2007). What Predicts Teachers' Attitudes Toward the Gifted? Gifted Child Quarterly, 51(3), 246-254. doi:10.1177/0016986207302719 Mendioroz, A., Gracia, P. y Aguilera, E. (2019). Una propuesta de formación docente para responder a las altas capacidades en la escuela inclusiva. Revista de Currículum y Formación del Profesorado, 23(1), 265-284.

Morrissey, M. L. (2006). Intervention training with a focus on differentiated curriculum and teachers' attitudes toward the gifted in regular elementary school classrooms. (Doctoral Dissertation), Columbia University Teachers College, New York, United States.

Ortega, P. (1986). La investigación en la formación de actitudes: problemas metodológicos y conceptuales. In Anales de pedagogía (4). 
Pacheco, F. (2002). Actitudes. Eúphoros, (5), 173-186.

Palomares, A. (2011). El modelo docente universitario y el uso de nuevas metodologías en la enseñanza, aprendizaje y evaluación. Revista de Educación, 355, 591-605.

Pegalajar, M. del C. y Colmenero, M. de J. (2017). Actitudes y formación docente hacia la inclusión en Educación Secundaria Obligatoria. Revista Electrónica de Investigación Educativa, 19(1), 84-97. doi:10.24320/redie.2017.19.1.765

Peña, M. J. y de la Poza, M. A. (2016). Similitudes y diferencias entre actitudes, estereotipos y prejuicios sociales. Avances de Investigación en Salud a lo largo del Ciclo Vital, 71, 77-91.

Pomar, C. Ma. (2001): La motivación de los superdotados en el contexto escolar. Santiago de Compostela: Universidad de Santiago de Compostela.

Polyzopoulou, K., Kokaridas, D., Patsiaouras, A. y Gari, A. (2014). Teachers' perceptions toward education of gifted children in Greek educational settings. Journal of Physical Education and Sport, 14(2), 211. doi:10.7752/jpes.2014.02033.

Plunkett, M. (2000). Impacting on teacher attitudes toward gifted students. Australasian Journal of Gifted Education, 9(2), 33-42.

Plunkett, M. y Kronborg, L. (2011). Learning to be a Teacher of the Gifted: The Importance of Examining Opinions and Challenging Misconceptions. Gifted and Talented International, 2(26), 31- 46.

Preckel, F., Baudson, TG, Krolak-Schwerdt, S. y Glock, S. (2015). ¿Dotados y desajustados? Actitudes implícitas y asociaciones automáticas relacionadas con niños superdotados. American Educational Research Journal, 5 (6),1160-1184. doi:10.3102/0002831215596413.

Polyzopoulou, K., Kokaridas, D., Patsiaouras, A. y Gari, A. (2014). Teachers' perceptions toward education of gifted children in Greek educational settings. Journal of Physical Education and Sport, 14(2), 211. doi:10.7752/jpes.2014.02033.

Reyes, L. (2007). La Teoría de la Acción Razonada. Implicaciones para el estudio de las actitudes. Investigación educativa duranguense, (7), 66-77.

Roa Bañuelos, M. D. M. (2017). Las altas capacidades intelectuales en el ámbito educativo de la Comunidad Autónoma del País Vasco. Creencias, valores y actitudes de docentes y estudiantes de educación (Tesis Doctoral). Universidad del País Vasco.

Sánchez, E. (1997): Hacia una didáctica para la educación de los niños superdotados". Revista Complutense de Educación. Madrid: Servicio de Publicaciones de la Universidad Complutense, 8(2), 57-70.

Seade, C., Encalada, G., Peñaherrera, M.J., Dávila, Y. y Vélez, X. (2019). ¿Puede la formación en altas capacidades afectar las actitudes de los maestros en Educación Primaria?. International Journal of Developmental and Educational Psychology. INFAD Revista de Psicología, 1(4), 441-450.

Tourón, J., Fernández, R. y Reyero, M. (2002). Actitudes del profesorado hacia la superdotación. Implicaciones para el desarrollo de programas de formación. Faisca 9, 95-110.

Toxclair, A. (2013). Preservice Teacher Attitudes Toward Giftedness. Roeper Review, 35(1), 58- 64. doi:10.1080/02783193.2013.740603

Trujillo, M.J., De la Poza, M.A. y Portero, N. (2016). Similitudes y diferencias entre actitudes, estereotipos y prejuicios sociales. En M.M. Molero, M.C. Pérez- Fuentes, J.J. Gázquez, A.B. Barragán, A. Martos, y M.M, Simón (Eds.). Avances de Investigación en Salud a lo largo del Ciclo Vital, (pp.71-16). ASUNIVEP. https://dialnet.unirioja.es/descarga/libro/677951.pdf\#page=72

Ubillos, S., Mayordomo, S., y Páez, D. (2004). Actitudes: Definición y Medición. Componentes de la Actitud. Modelo de la Acción Razonda y Acción Planificada. Psicología social, cultura y educación

Verdugo, M., Arias, B. y Jenaro, C. (1994). Actitudes hacia las personas con minusvalía. Madrid: Inserso

Vilaseca, G. (2006). Actitud y práctica educativa del profesorado hacia las necesidades educativas especiales de superdotación. En I Jornadas Nacionales sobre escuela y altas capacidades. Intercambio de experiencias (pp. 11-126). Universidad de Barcelona, España 
ACTITUDES Y CREENCIAS DE LOS DOCENTES HACIA EL ALUMNADO CON ALTAS CAPACIDADES INTELECTUALES Y

LA IMPORTANCIA DE LA FORMACIÓN DEL PROFESORADO EN ESTE ÁMBITO

Watts, G. (2006). Teacher attitudes to the acceleration of the gifted: A case study from New Zealand gifted and talented. Journal of the National Association for Gifted Children, 10 (1), 11-19. 